\title{
Influenza vaccine effectiveness in adults 65 years and older, Denmark, 2015/16 - a rapid epidemiological and virological assessment
}

H Emborg ${ }^{1}$, TG Krause ${ }^{1}$, L Nielsen ${ }^{2}$, MK Thomsen ${ }^{3}$, CB Christiansen ${ }^{4}$, MN Skov ${ }^{5}$, XC Nielsen ${ }^{6}$, LS Weinreich ${ }^{7}$, TK Fischer ${ }^{8}$, J Rønn ${ }^{8}$, R Trebbien ${ }^{8}$

1. Department of Infectious Disease Epidemiology, Statens Serum Institut, Copenhagen, Denmark

2. Department of Clinical Microbiology, Herlev Hospital, Herlev, Denmark

3. Department of Clinical Microbiology, Aarhus University Hospital, Aarhus, Denmark

4. Department of Clinical Microbiology, Rigshospitalet, Copenhagen, Denmark

5. Department of Clinical Microbiology, Odense University Hospital, Odense C, Denmark

6. Department of Clinical Microbiology, Slagelse Hospital, Slagelse, Denmark

7. Department of Clinical Microbiology, Aalborg University Hospital, Aalborg, Denmark

8. Department of Microbiological Diagnostics and Virology, National Influenza Center, Statens Serum Institut, Copenhagen, Denmark

Correspondence: Hanne-Dorthe Emborg (hde@ssi.dk)

Citation style for this article:

Emborg H, Krause TG, Nielsen L, Thomsen MK, Christiansen CB, Skov MN, Nielsen XC, Weinreich LS, Fischer TK, Rønn J, Trebbien R. Influenza vaccine effectiveness in adults 65 years and older, Denmark, 2015/16 - a rapid epidemiological and virological assessment. Euro Surveill. 2016;21(14):pii=30189. DOI: http://dx.doi. org/10.2807/1560-7917.ES.2016.21.14.30189

In Denmark, both influenza $\mathrm{A}\left(\mathrm{H}_{1} \mathrm{~N}_{1}\right)$ pdmog and influenza $B$ co-circulated in the $2015 / 16$ season. We estimated the vaccine effectiveness (VE) of the trivalent influenza vaccine in patients 65 years and older using the test-negative case-control design. The adjusted VE against influenza $\mathrm{A}\left(\mathrm{H}_{1} \mathrm{~N}_{1}\right)$ pdmog was $35.0 \%(95 \%$ confidence interval $(\mathrm{Cl}): 11.1-52.4)$ and against influenza B $4.1 \%(95 \% \mathrm{Cl}:-22.0$ to 24.7$)$. The majority of influenza $A\left(\mathrm{H}_{1} \mathrm{~N}_{1}\right)$ pdmog circulating in 2015/16 belonged to the new genetic subgroup subclade 6B.1.

In Denmark, both influenza $\mathrm{A}\left(\mathrm{H}_{1} \mathrm{~N}_{1}\right)$ pdmog and influenza B co-circulated in the $2015 / 16$ season. The trivalent influenza vaccine (TIV) did not include the circulating influenza B Victoria lineage and there is evidence in Europe for genetic evolution of the circulating influenza $\mathrm{A}\left(\mathrm{H}_{1} \mathrm{~N}_{1}\right)$ pdmog virus [1]. We estimated the influenza vaccine effectiveness (VE) in people aged 65 years and older. In addition, we describe the genetic and antigenic characteristics of the influenza $A\left(\mathrm{H}_{1} \mathrm{~N}_{1}\right)$ pdmog variant and the influenza $B$ strain circulating in Denmark.

\section{Data for vaccine effectiveness estimation}

In the Danish Microbiology Database, all patients swabbed at the general practitioner's (GP) or at hospital and tested for influenza $A$ and $B$ viruses by PCR are registered in real time [2]. During the influenza season, national guidelines recommend that patients belonging to risk groups, including the elderly who present with influenza symptoms at GPs and hospitals are swabbed and tested for influenza. At hospitals, all patients with lower respiratory infections are also recommended to be swabbed. All diagnostic influenza tests from patients aged 65 years and older were included in this study.

Influenza symptoms were defined as sudden onset of fever, muscle ache and upper airway symptoms. The trivalent influenza vaccine (TIV) is offered free of charge to Danish citizens 65 and older between week 40 and week 53, and date of vaccination is registered in the Danish Vaccination Register [3]. In The Danish National Hospital Register, data on all hospital admissions are collected [4]. Comorbidities that can lead to severe influenza disease and were diagnosed between October 2010 and October 2015 were extracted from the Danish National Hospital Register.

Data from the Danish Microbiology Database, the Danish Vaccination Register and the Danish National Hospital Register were linked using unique identifiers.

\section{Case definitions and statistical analysis}

Cases were defined as patients who tested positive for influenza $A\left(\mathrm{H}_{1} \mathrm{~N}_{1}\right)$ pdmog or influenza $B$, and a patient was only included the first time a test was positive for either type. Controls were patients who tested negative for both influenza A and B. Patients were considered vaccinated if they had received the TIV at least two weeks before the sample was taken. A logistic regression model was used to estimate VE against influenza $A\left(\mathrm{H}_{1} \mathrm{~N}_{1}\right)$ pdmog and influenza $B$ using the test-negative case-control design (1-OR) $\times 100 \%$. The 
Trivalent influenza vaccines received $(\mathrm{n}=1,831)$ and laboratory-confirmed influenza A(H1N1)pdm09 and B cases among tested patients $\geq 65$ years $(n=468)$, Denmark, 28 September 2015-9 March 2016

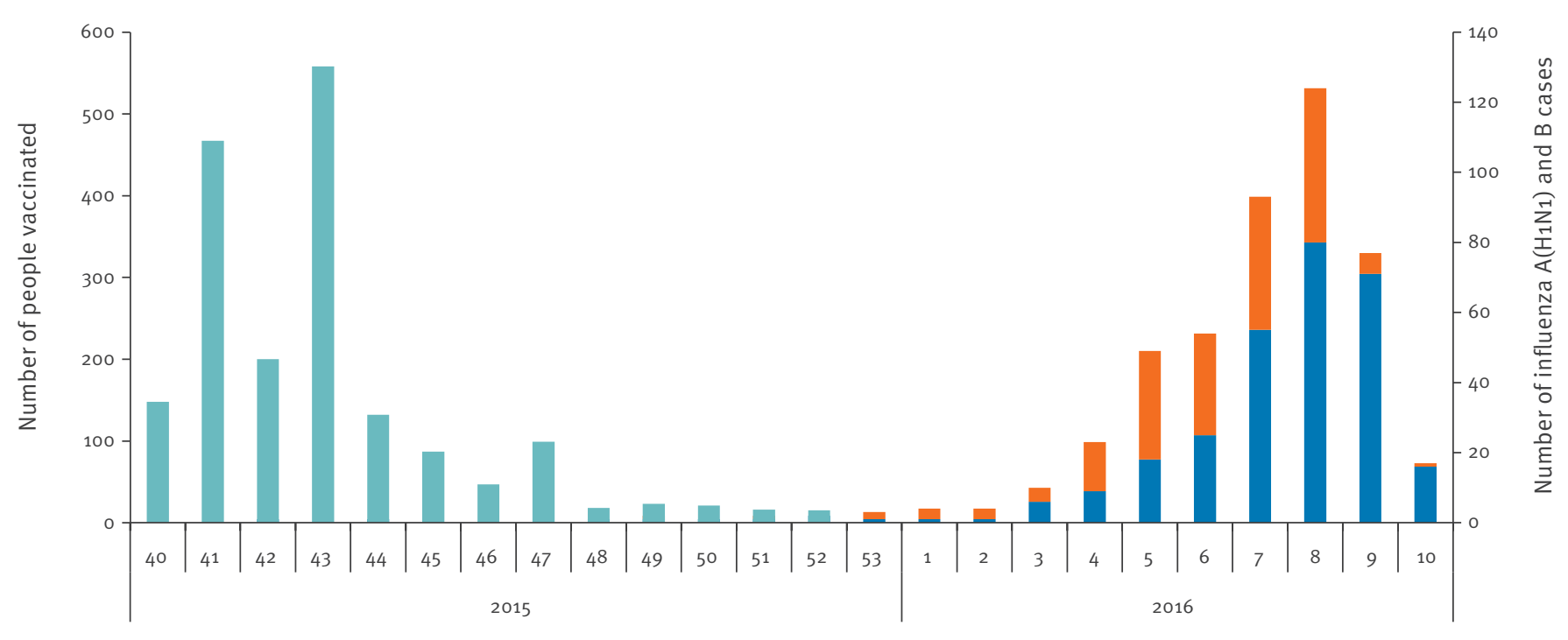

Date (epidemiological weeks)

Seasonal influenza vaccines 2015/16 Influenza A(H1N1)pdmo9 Influenza B

Influenza vaccines are given free of charge to the elderly 65 years and older from 1 October to 31 December. Due to delay in registration of vaccinations, data from week 53 were not available at the time this analysis was performed.

In weeks 40 to 53 , between o and two influenza $A\left(\mathrm{H}_{1} \mathrm{~N}_{1}\right)$ and $\mathrm{B}$ cases were registered per week (not visible at presented range of the $y$-axis).

estimates were adjusted for sex and co-morbidities diagnosed within a five-year period before the 2015/16 influenza season. Among 195 subtyped influenza A isolates from patients aged 65 years and older, less than $10 \%(n=18)$ were $A\left(H_{3} N_{2}\right)$ and $V E$ against this subtype was not estimated.

The statistical programme SAS version 9.4 was used for the descriptive and statistical analyses (SAS Institute, Cary, United States).

\section{Influenza virus characterisation}

All influenza samples received at The National Influenza Center in Denmark (NIC) were screened for influenza virus by an in-house multiplex real-time reverse-transcriptase PCR (qRT-PCR), with primers and probes detecting influenza $A$ and $B$ virus as well as subtypes of $\mathrm{H}_{3}$ haemagglutinin (HA) and N1pdmo9 neuraminidase. Subtyping of influenza $B$ virus is also performed by an in-house duplex qRT-PCR which differentiates between the Yamagata and Victoria lineage on a fragment of the HA gene.

Sequencing of the HA gene of influenza $A\left(\mathrm{H}_{1} \mathrm{~N}_{1}\right)$ pdmog and influenza $B$ viruses was performed on extracted viral RNA from 62 and 20 samples, respectively. Total nucleic acid was extracted using $200 \mu \mathrm{l}$ of sample material and the MagNA Pure LC Total Nucleic Acid Isolation Kit on the MagNa Pure 96/32 (Roche). RT-PCR of the complete HA gene was performed using inhouse primers and an in-house one-step RT-PCR programme on a TRIO cycler (Biometra). Sequencing was performed by using Big Dye chemistry on an $\mathrm{AB} 3500$ capillary sequencer (Thermo Fisher). Assembly of contigs was done in Bionumerics version 6.6 (Applied maths) and alignment and phylogenetic analysis were conducted with MEGA version 6 [5]. For alignment, the Muscle algorithm was used and phylogenetic trees were created by the maximum likelihood method using 1,000 bootstrap replicates. Sequences were also analysed by BLAST at NCBI GenBank, the Global Initiative on Sharing All Influenza Data (GISAID) and at the FLUSERVER [6]. The authors gratefully acknowledge the 59 originating and submitting laboratories who contributed sequences used in the phylogenetic analysis to GISAID (www.gisaid.org).

Virus isolation was successful for 32 influenza $A\left(\mathrm{H}_{1} \mathrm{~N}_{1}\right)$ pdmog and 13 influenza $B$ samples by standard procedures in confluent monolayers of MDCK and/or MDCKSIAT cells [7]. Several samples were shipped in E-swab medium which is cytotoxic and therefore is challenging for virus isolation [8]. Antigenic characterisation was performed by $\mathrm{HA}$ inhibition ( $\mathrm{HAl}$ ) test [7] using reference ferret antiserum against $A / C a$ lifornia/07/2009 (H1N1pdmo9), B/Brisbane/60/2008 (Victoria lineage) and $\mathrm{B} /$ Phuket/3073/2013 (Yamagata lineage) provided 


\section{FIGURE 2}

Phylogenetic tree of the haemagglutinin gene with reference viruses for the different phylogenetic clades of H1N1pdm09 influenza A viruses $(\mathrm{n}=40)$

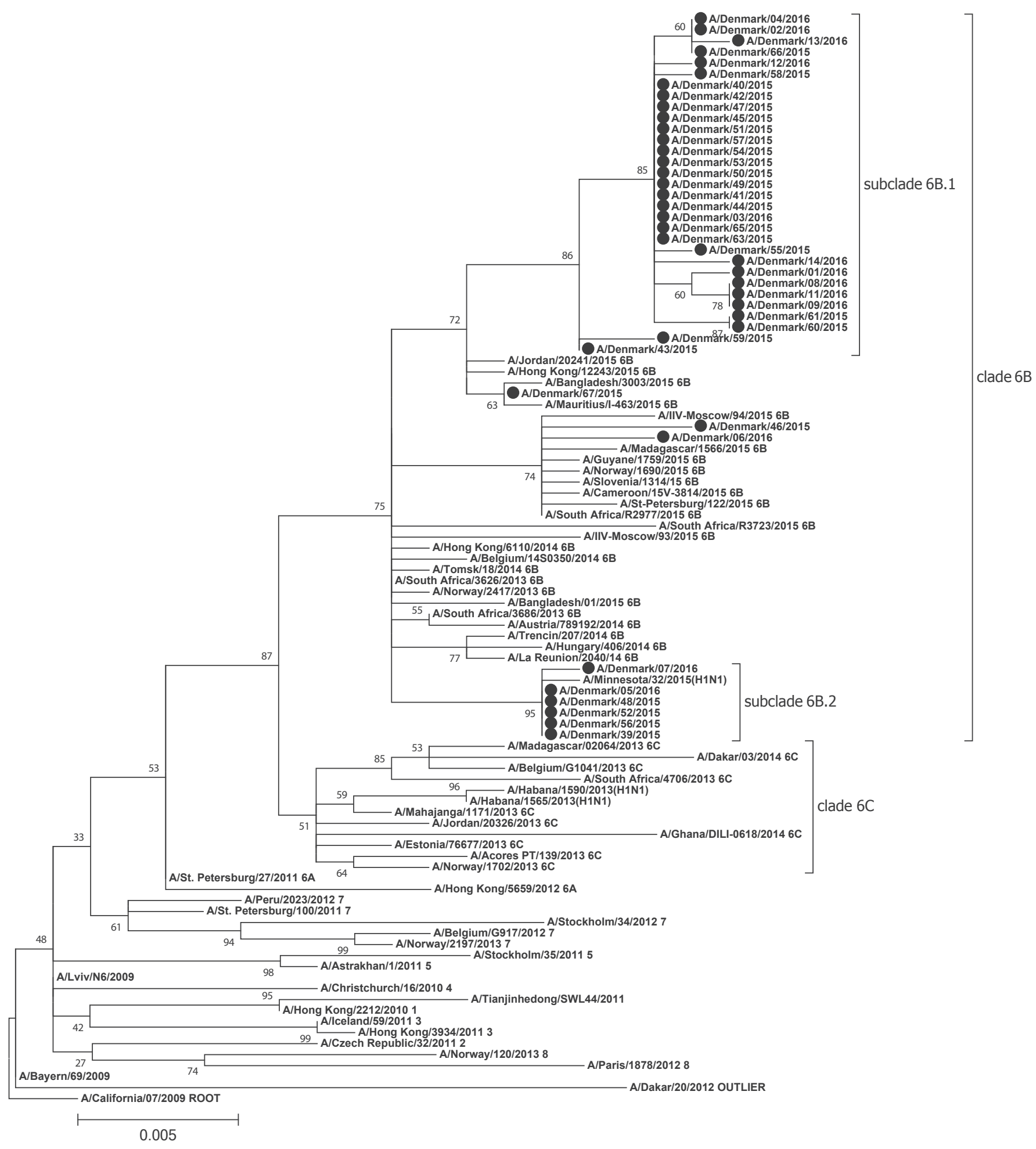

The Danish viruses are indicated with a black circle. A subclade formed by viruses with the amino acid substitutions S101N, S179N and I233T, subclade 6B.1, is indicated as well as the subclade formed by viruses with the V169T, V190l, E508G and D518E substitutions, subclade 6B.2. The authors gratefully acknowledge the 59 originating and submitting laboratories who contributed sequences used in the phylogenetic analysis to GISAID (www.gisaid.org). 
Laboratory-confirmed influenza A(H1N1)pdm09 and B cases $(\mathrm{n}=468)$ and influenza A and B test-negative controls $(n=3,363)$ aged $\geq 65$ years by trivalent influenza vaccination status, age group and sex, and vaccination coverage among influenza cases and controls by age group and sex, Denmark, 28 September 2015-9 March 2016

\begin{tabular}{|c|c|c|c|c|c|c|c|c|c|}
\hline \multirow[b]{2}{*}{ Characteristic } & \multicolumn{3}{|c|}{ Influenza $\mathrm{A}\left(\mathrm{H}_{1} \mathrm{~N}_{1}\right)$ pdmo9 } & \multicolumn{3}{|c|}{ Influenza B } & \multicolumn{3}{|c|}{ Controls } \\
\hline & $\begin{array}{c}\text { Vaccinated } \\
\text { (n) }\end{array}$ & $\begin{array}{c}\text { Not } \\
\text { vaccinated } \\
(\mathrm{n})\end{array}$ & $\begin{array}{l}\text { Vaccination } \\
\text { coverage } \\
(\%)\end{array}$ & $\begin{array}{c}\text { Vaccinated } \\
\text { (n) }\end{array}$ & $\begin{array}{c}\text { Not } \\
\text { vaccinated } \\
(n)\end{array}$ & $\begin{array}{l}\text { Vaccination } \\
\text { coverage } \\
(\%)\end{array}$ & $\begin{array}{l}\text { Vaccinated } \\
\text { (n) }\end{array}$ & \begin{tabular}{|c|} 
Not \\
vaccinated \\
(n)
\end{tabular} & $\begin{array}{l}\text { Vaccination } \\
\text { coverage (\%) }\end{array}$ \\
\hline \multicolumn{10}{|l|}{ Age group } \\
\hline $65-69$ & 16 & 42 & 27.6 & 37 & 42 & 46.8 & 337 & 488 & 40.8 \\
\hline $70-74$ & 20 & 29 & 40.8 & 37 & 41 & 47.4 & 385 & 458 & $45 \cdot 7$ \\
\hline $75-79$ & 18 & 22 & 45.0 & 27 & 34 & $44 \cdot 3$ & 363 & 323 & 52.9 \\
\hline$\geq 80$ & 13 & 17 & 43.3 & 34 & 39 & 46.6 & 544 & 466 & 53.9 \\
\hline \multicolumn{10}{|l|}{ Comorbidities } \\
\hline No & 15 & 34 & 30.6 & 36 & 66 & $35 \cdot 3$ & 347 & 480 & 42.0 \\
\hline Yes & 52 & 76 & 40.6 & 99 & 90 & 52.4 & 1,282 & 1,255 & 50.5 \\
\hline \multicolumn{10}{|l|}{ Sex } \\
\hline Female & 28 & 45 & 38.4 & 70 & 79 & 50.0 & 780 & 865 & 47.4 \\
\hline Male & 39 & 65 & 37.5 & 65 & 77 & 45.8 & 849 & 869 & 49.4 \\
\hline Total & 67 & 110 & 37.8 & 135 & 156 & 46.4 & 1,629 & $1,734^{a}$ & 48.3 \\
\hline
\end{tabular}

a Sex was not known for one person.

by the World Health Organization (WHO) Collaboration Centre, Mill Hill, London.

\section{Vaccine effectiveness results}

By 9 March 2016, 3,831 patients 65 years and older were tested for influenza $A\left(\mathrm{H}_{1} \mathrm{~N}_{1}\right)$ pdmog and $B$, and $65 \%$ of them were swabbed at a hospital. In total, 177 patients were positive for influenza $A\left(\mathrm{H}_{1} \mathrm{~N}_{1}\right)$ pdmog and 291 for influenza B. In total, 1,505 (82\%) of 1,831 study participants had received the TIV before 2 November in 2015 (Figure 1).

Vaccine coverage in cases diagnosed with influenza $\mathrm{A}\left(\mathrm{H}_{1} \mathrm{~N}_{1}\right)$ pdmog was $37.8 \%$, which is lower than the coverage in controls (48.3\%), cases diagnosed with influenza B (46.4\%) (Table) and the estimated national coverage of $44 \%$ (data not shown). The coverage, for both cases and controls, was higher among patients with comorbidities compared with patients without comorbidities (Table).

Adjusted interim VE among those aged 65 years and older against influenza $\mathrm{A}\left(\mathrm{H}_{1} \mathrm{~N}_{1}\right)$ pdmog was $35.0 \%$ (95\% confidence interval $(\mathrm{Cl}): 11.1-52.4$ ) and against influenza B 4.1\% (95\% Cl: -22.0 to 24.7$)$.

\section{Virus characterisation results}

Full gene sequencing of the HA gene from 62 influenza $A\left(\mathrm{H}_{1} \mathrm{~N}_{1}\right)$ pdmog samples revealed in 46 of them an amino acid substitution at position 179 ( $\mathrm{H}_{1}$ complete open reading frame numbering) from serine to asparagine, which leads to a potential glycosylation site formed by positions 179-181 with the amino acid motif asparagine-glutamine-serine (NQS) (Table).
Additional substitutions were revealed at amino acid position $\mathrm{S}_{101} \mathrm{~N}$ and $\mathrm{I}_{233 \mathrm{~T}}$ in the 46 samples having the S179N. Two of the patient samples had an additional substitution at $\mathrm{H}_{155} \mathrm{Y}$. Nine samples had a different amino acid motif with substitutions at positions $\mathrm{V}_{16} \mathrm{~T}$, V190l, E508G and D518E.

Phylogentic analysis revealed that all 62 sequenced $\mathrm{HA}$ genes of $A\left(\mathrm{H}_{1} \mathrm{~N}_{1}\right)$ pdmog viruses belonged to genetic clade 6B (Figure 2), however, the 46 viruses with the S101N, S179N, and I233T substitutions formed their own subclade which now is categorised by the WHO as subclade 6B.1. In addition, the nine $\mathrm{V}_{169} \mathrm{~T}, \mathrm{~V} 190 \mathrm{l}$, $\mathrm{E}_{508 \mathrm{G}}$ and $\mathrm{D}_{518 \mathrm{E}}$ viruses clustered together with the A/Minnesota/32/2015(H1N1pdmo9) virus (Figure 2) and are now categorised as subclade 6B.2.

Of the $32 \mathrm{~A}\left(\mathrm{H}_{1} \mathrm{~N}_{1}\right)$ pdmog viruses isolated in cell culture, 25 belonged to subclade 6B.1, three belonged to subclade 6B.2, and four belonged to clade 6B. Antigenic characterisation showed all 32 virus isolates to be equally inhibited or inhibited to a lesser extent (twoto fourfold decrease in $\mathrm{HAl}$ titre), by ferret antiserum against $A / C a l i f o r n i a / 07 / 2009$ (H1N1pdmo9) compared with the A/California/07/2009 (H1N1pdmo9) reference virus $\mathrm{HAl}$ titres.

Of 447 influenza B virus samples from all age groups received for the national influenza surveillance programme at NIC Denmark by mid-March 2016, 350 were subtyped; 307 (88\%) belonged to the B-Victoria lineage and $43(12 \%)$ belonged to the B-Yamagata lineage. The HA genes of $15 \mathrm{~B}$-Victoria viruses were sequenced and all belonged to clade $1 \mathrm{~A}$, corresponding to the 
strain included in the quadrivalent vaccine but not included in the trivalent vaccine used in Denmark in the current season. Antigenic characterisation by $\mathrm{HAl}$ test of 13 virus isolates showed a two- to fourfold decrease in $\mathrm{HAl}$-titre using the ferret antiserum against $\mathrm{B} /$ Brisbane/60/2008 compared with the vaccine reference virus $B /$ Brisbane/60/2008. None of the B-Victoria viruses was inhibited by the $B$-Yamagata reference antiserum B/Phuket/3073/2013.

\section{Discussion}

Due to the late start of the influenza season in Europe only few interim VE estimates have been published $[9,10]$ and in particular, little information is available on the VE in those aged 65 years and older, an important target group for influenza vaccination. Furthermore, a mismatch was observed between the circulating B-Victoria lineage and the B-Yamagata lineage included in the TIV for the northern hemisphere.

We found no effect of the TIV against influenza B 4.1\% ( $95 \% \mathrm{Cl}:-22.0$ to 24.7 ), which accounted for $62 \%$ of the influenza detections in patients aged 65 years and older in Denmark until 9 March 2016. This can be explained by the mismatch because $88 \%$ of the $B$ infections were Victoria lineage. This is in line with findings from Hong Kong in 2011/12 where B-Victoria was included in the vaccine and VE against paediatric influenza B-Yamagata hospitalisation was estimated at $9.5 \%(95 \% \mathrm{Cl}:-240.4$ to 76.0$)$ [11]. However, in the same season, a study from the United States estimated a VE of $66 \%(95 \% \mathrm{Cl}: 38-81)$ against B-Yamagata although only the B-Victoria lineage was included in the vaccine [12], which could suggest cross-protection between lineages. Antigenic characterisation at the Danish NIC supports a lack of cross-reactivity between B-Yamagata and B-Victoria when using the current season's vaccine antiserum against $B /$ Brisbane/60/2008 and B/Phuket/3073/2013 in the HAl test which is also reported in the study from Hong Kong [11]. Influenza $B$ lineage-specific TIV VEs have earlier been estimated in seasons with both mismatch and/or cocirculation of two influenza B lineages. Some VE studies have suggested cross-protection between lineages and others not. The reasons for these differences are not known but may be explained by methodological issues or by differences in population immunity due to variations in vaccination strategies or differences in circulating lineages between regions [13].

It is likely that immunity against influenza B Victoria in the Danish population is low, as only few isolates from this lineage have been detected in Denmark since 2010/11 and have not been included in the vaccine since 2011/12. Influenza B-Victoria also dominates over B-Yamagata in the rest of Europe [14], and if the quadrivalent vaccine had been used instead of TIV during the current season morbidity due to influenza B might have been lower.
We found a moderate to low VE against influenza $\mathrm{A}\left(\mathrm{H}_{1} \mathrm{~N}_{1}\right)$ pdmog of $35.0 \%(95 \% \mathrm{Cl}: 11.1-52.4)$ in patients aged 65 years and older, although the majority of influenza $A\left(\mathrm{H}_{1} \mathrm{~N}_{1}\right)$ pdmog circulating in Denmark in the $2015 / 16$ season belonged to the new genetic subclade 6B.1. VE against influenza $A\left(\mathrm{H}_{1} \mathrm{~N}_{1}\right)$ pdmog in the current season was similar to the VE against influenza $A\left(\mathrm{H}_{1} \mathrm{~N}_{1}\right)$ pdmog in the $2014 / 15$ season in Denmark of $31 \%(95 \%$ $\mathrm{Cl}$ : -0.7 to 52.7$)$ where 114 patients were positive for influenza $\mathrm{A}\left(\mathrm{H}_{1} \mathrm{~N}_{1}\right)$ pdmog and 3,351 patients tested negative (data not shown). This estimate also corresponds to the estimated VE of $22 \%$ ( $95 \% \mathrm{Cl}:-44.4$ to 58.4) against influenza $A\left(\mathrm{H}_{1} \mathrm{~N}_{1}\right)$ pdmog in the same age group in season 2014/15 reported by I-Move following a multicentre case-control study [15].

\section{Conclusion}

We estimated similar VE against influenza $A\left(\mathrm{H}_{1} \mathrm{~N}_{1}\right)$ pdmog in season 2014/15 and 2015/16 in those aged 65 years and older in spite of the occurrence of the new subclade 6B.1. This is reassuring as the WHO recommendations for the influenza $A\left(\mathrm{H}_{1} \mathrm{~N}_{1}\right)$ pdmog component in the $2016 / 17$ vaccine for the northern hemisphere remained the same as in previous years, while the influenza B component changed from Yamagata to Victoria [16].

\section{Acknowledgement}

Test results for influenza virus were obtained from the Danish Microbiology Database (MiBa, http://miba.ssi.dk), which contains all electronic reports from departments of clinical microbiology in Denmark since 2010, and we acknowledge the collaboration with the MiBa Board of Representatives.

The authors gratefully acknowledge the 59 originating and submitting laboratories who contributed sequences used in the phylogenetic analysis to GISAID (www.gisaid.org).

We also acknowledge for the laboratory work, Dennis Jelsbak Schmidt and Bente Andersen, National Influenza Center Denmark, Statens Serum Institut, Copenhagen, Denmark.

Statens Serum Institut would also like to acknowledge the participation in the I-MOVE+ (Integrated Monitoring of Vaccines in Europe) project that has received funding from the European Union's Horizon 2020 research and innovation programme under grant agreement № 634446 .

Conflict of interest

None declared.

Authors' contributions

Hanne-Dorthe Emborg led the writing of the paper. Ramona Trebbien was responsible for the virological characterization and Jesper Rønn for the laboratory work. Lene Nielsen, Marianne Kragh Thomsen, Claus Bohn Christiansen, Marianne Nielsine Skov, Xiaohui Chen Nielsen and Lenette Sandborg Weinreich performed the initial diagnostics of influenza positive samples. Tyra Grove Krause and Thea Kølsen Fischer conceptualised the study together with Hanne-Dorthe Emborg and Ramona Trebbien and discussed 
the data and perspectives. All authors provided contributions to the paper and approved the final version.

\section{References}

1. European Centre for Disease Prevention and Control (ECDC) Seasonal influenza 2015-2016 in the EU/EEA countries. Risk assessment. Stockholm: ECDC; 2016. Available from: http:// ecdc.europa.eu/en/publications/Publications/seasonalinfluenza-risk-assessment-2015-2016.pdf

2. MiBa Board of Representatives,Voldstedlund M, Haarh M, Mølbak K. The Danish Microbiology Database (MiBa) 2010 to 2013.Euro Surveill. 2014;19(1):20667. DOI: 10.2807/1560-7917. ES2014.19.1.20667 PMID: 24434175

3. Grove Krause T, Jakobsen S, Haarh M, Mølbak K. The Danish vaccination register.Euro Surveill. 2012;17(17):20155.PMID: 22551494

4. Andersen TF, Madsen M, Jørgensen J, Mellemkjoer L, Olsen JH. The Danish National Hospital Register. A valuable source of data for modern health sciences.Dan Med Bull. 1999;46(3):263-8.PMID: 10421985

5. Tamura K, Stecher G, Peterson D, Filipski A, Kumar S. MEGA6: Molecular Evolutionary Genetics Analysis version 6.0. Mol Bio Evol. 2013;30(12):2725-9. DOI: 10.1093/molbev/mst197 PMID: 24132122

6. FluSurver. Influenza surveillance - prepared for next wave. Singapore; A*STAR Bioinformatics Institute. [Accessed: 14 Mar 2016]. Available from: http://flusurver.bii.a-star.edu.sg/

7. World Health Organization (WHO) Global Influenza Surveillance Network. Manual for the laboratory diagnosis and virological surveillance of influenza. Geneva: WHO; 2011. Available from: http://apps.who.int/iris/ bitstream/10665/44518/1/9789241548090_eng.pdf

8. Trebbien R, Andersen B, Rønn J, McCauley J, Fischer TK. ESwab challenges influenza virus propagation in cell cultures. Euro Surveill. 2014;19(50):20995. DOI: 10.2807/1560-7917. ES2014.19.50.20995 PMID: 25597539

9. Kissling $\mathrm{E}$, Valenciano $\mathrm{M}$. Early influenza vaccine effectiveness results 2015-16: I-MOVE multicentre case-control study. Euro Surveill. 2016;21(6):30134. DOI: 10.2807/1560-7917. ES.2016.21.6.30134 PMID: 26898240

10. Pebody R, Warburton F, Ellis J, Andrews N, Potts A, Cottrell $\mathrm{S}$, et al. Effectiveness of seasonal influenza vaccine in preventing laboratory-confirmed influenza in primary care in the United Kingdom: 2015/16 mid-season results. Euro Surveill. 2016;21(13):30179.

11. Chiu SS, Feng S, Chan KH, Lo JY, Chan EL, So LY, et al. Hospitalbased vaccine effectiveness against influenza $B$ lineages, Hong Kong, 2009-14. Vaccine. 2016;pii: So264-410X(16)30005-6

12. Ohmit SE, Thompson MG, Petrie JG, Thaker SN, Jackson ML, Belongia EA, et al. Influenza vaccine effectiveness in the 2011-2012 season: protection against each circulating virus and the effect of prior vaccination on estimates. Clin Infect Dis. 2014;58(3):319-27. DOI: 10.1093/cid/cit736 PMID: 24235265

13. Skowronski DM, Janjua NZ, Sabaiduc S, De Serres G, Winter AL, Gubbay JB, et al. Influenza A/subtype and B/lineage effectiveness estimates for the 2011-2012 trivalent vaccine: cross-season and cross-lineage protection with unchanged vaccine. J Infect Dis. 2014;210(1):126-37. DOI: 10.1093/infdis/ jiuo48 PMID: 24446529

14. European Centre for Disease Prevention and Control (ECDC). Influenza situation in week 9 2016. Stockholm: ECDC; 2016. Available from: http://ecdc.europa.eu/en/healthtopics/ seasonal_influenza/epidemiological_data/Pages/Latest surveillance_data.aspx

15. Valenciano M, Kissling E, Reuss A, Rizzo C, Gherasim A, Horváth JK, et al. Vaccine effectiveness in preventing laboratory-confirmed influenza in primary care patients in a season of co-circulation of influenza $A\left(\mathrm{H}_{1} \mathrm{~N}_{1}\right)$ pdmog, $B$ and drifted $A\left(\mathrm{H}_{3} \mathrm{~N}_{2}\right)$, I-MOVE Multicentre Case-Control Study, Europe 2014/15. Euro Surveill. 2016;21(7):30139. DOI: 10.2807/1560-7917.ES.2016.21.7.30139 PMID: 26924024

16. Eurosurveillance editorial team., WHO recommendations on the composition of the $2016 / 17$ influenza virus vaccines in the northern hemisphere. Euro Surveill. 2016;21(8):30147. credit to the source, provide a link to the licence, and indicate if changes were made.

This article is copyright of the authors, 2016.

\section{License and copyright}

This is an open-access article distributed under the terms of the Creative Commons Attribution (CC BY 4.0) Licence. You may share and adapt the material, but must give appropriate 